\title{
Expressionismus - europäische Moderne auf deutsch oder deutsche Seelenkrankheit? Bemerkungen zur Forschung
}

\section{RICHARD BRINKMANN}

Der Begriff 'Expressionismus', seine Bedeutung, sein Geltungsbereich, ist immer noch eine Plage. Immer noch beginnen viele Studien zu irgendeinem Gegenstand, der damit zu tun hat oder zu tun haben könnte, mit dem Seufzer, daß gar nicht feststehe, was Expressionismus eigentlich sei. Nur wenige ergreifen sozusagen die Flucht nach vorwärts und nennen - manchmal geradezu auftrumpfend - tautologisch expressionistisch, was sie eben expressionistisch nennen. Zuweilen resigniert man aber überhaupt vor der Aufgabe, einen Kollektivbegriff dieses Namens zu definieren und zu füllen und ihm konkrete Realisate zuzuordnen: das sei 'philologische Anmaßung' von 'Literaturprofessoren', meint einer, der über die Untugenden dieser Kaste erhaben zu sein scheint. Festzustellen, "wer einst zum Expressionismus gehört hatte und wer nicht", dies seien 'Verirrungen der Germanistik'. So steht es in einem überaus gründlichen und materialreichen, mit großer Hingebung geschriebenen neueren Buch mit dem lapidaren Titel Der Expressionismus. Verfasser ist Wolfgang Rothe. ${ }^{1}$ Auch da wird offenbar mit Wasser gekocht: es gibt Expressionismus, und es gibt Leute, mit deren Äußerungen man belegen kann, was dieser Expressionismus ist, die also - irgendwie - dazu gehört haben, und es gibt andere - auch das ist ziemlich logisch--, die eben nicht 'dazu gehört' haben und die daher zum Beleg einer Wesensbestimmung nicht taugen. An den hermeneutischen Zirkel mag man dabei erinnern; weit ausbreiten und erörtern müssen wir das Problem hier nicht. Seine Tücke ist jedem Historiker geläufig, der sich mit Epochenproblemen herumschlägt. Einen kleinen Augenblick sollten wir aber doch wenigstens eine Seite der Frage reflektieren, was es mit Epochenbegriffen, Epochennamen auf sich hat, wie sie zustande kommen und was sie leisten können. Einen Moment Theorie also. Auffallend ist zunächst, daß Epochennamen, wenn es sie einmal gibt, kaum mehr abzuschaffen und zum Verschwinden zu bringen sind. Den Geist 'Epochennamen' (oder auch 'Stileinheitsbegriff'), einmal gerufen, wird kein geschichtsschreibender Zauberlehrling mehr los. Das eben apostrophierte Beispiel ist nur eines unter zahllosen für die Tatsache, daß der Expressionismus-Begriff lebt auch da, wo man ihn für tot, für falsch, für unangemessen, unbrauchbar, ungehörig erklärt. Obgleich fast jeder, der auf sich hält und den guten Ton kennt, sich zur Skepsis gegenüber dem Namen 
Expressionismus verpflichtet glaubt und auch andere dazu aufruft, hat der Begriff sich in der Forschung bis auf den gegenwärtigen Tag doch geradezu überwältigend etabliert und bestätigt - so vielfältig auch die Bedeutungsnuancen sein mögen, so beträchtlich auch die Differenzen in der Feststellung der zeitlichen Grenzen. Woher bezieht denn nun so ein Epochenbegriff seinen Gehalt? 2 Er hat ihn - schlicht gesagt - nirgendwo anders her als von Gnaden der Wissenschaft, in unserem Fall: der Kunst- und Literarhistorie. Aus vielerlei Einzelbeobachtungen, Einzelbeschreibungen und -interpretationen reichert sich ein solcher Begriff an, schließt sich in einem Wort zusammen, das an sich zunächst ziemlich zufällig und gleichgültig sein mag. Es ist zum Beispiel nicht einmal so schrecklich wichtig, ob der Bericht über den angeblich ersten Gebrauch des Terminus 'Expressionismus' (jeder Enzyklopädieartikel muß natürlich damit anfangen) authentisch ist oder nicht. Der Gehalt des Begriffs, dessen Bildung indessen einen keineswegs beliebigen und verzichtbaren Akt des Verstehens bedeutet, ist am Ende mehr als die Summe der zahlreichen Einzelphänomene, die in ihm befaßt sind. Wie er gleichwohl aus der Beobachtung der historischen Einzelphänomene entsteht, liefert er seinerseits wiederum Kriterien für die Interpretation und das Verstehen dieser Einzelheiten, die ihn begründet haben. In immer erneutem Umgang mit dem Einzelnen, im immer erneuten Erschließen und Beschreiben von konkreten Einzelphänomenen mag sich die gehaltvolle Abstraktion des Epochen- oder Stilrichtungsbegriffs modifizieren, anreichern, differenzieren. Seinen wissenschaftsgeschichtlichen Gehalt kann er nicht mehr verlieren oder verleugnen, wenn man ihn nicht abschafft - und das, wie gesagt, hat wenig Aussicht auf Erfolg. Es ist kein Zufall, daß sich alle Versuche, Namen solcher Art total neu zu fassen, ihren bisherigen Gehalt vollkommen zu vergessen, damit sie einen angeblich richtigeren, ganz anderen repräsentieren, auch dann als verfehlt und folgenlos erwiesen haben, wenn die Vorschläge von höchst sachkundigen und kompetenten Leuten kamen. Beispiele dafür kennt jeder: also etwa statt vom Barock nur noch vom siebzehnten Jahrhundert zu sprechen, statt vom Biedermeier von 'Bürgerklassik'. Man wird auch den synthetischen Charakter des Kollektivbegriffs für Namen von Epochen oder Stilrichtungen anerkennen und akzeptieren müssen. Die Reduzierung auf Elemente und Einzelschritte der Entstehung des Begriffs kann aufschlußreich sein und ist nicht überflüssig. Aber erstaunlicherweise - oder auch gar nicht erstaunlicherweise führt das nicht zu einem Umriß des Namens in seinem gegenwärtigen Gebrauch und um das geht es schließlich -, sondern eher zu seiner Auflösung. Musterbeispiele dafür sind die glänzenden Studien René Welleks zu den Begriffen 'Romantik', 'Barock', 'Realismus'. 3 Da wird jeweils mit einer stupenden Gelehrsamkeit eine verwirrende Fülle von Details, von Partialgenesen und -kausalitäten ausgebreitet, die zu dem Epochenbegriff geführt haben. Wo kommt das Wort wann in welchem Sinne im weitesten Bereich der Epoche vor, die es benennt? Das ist da die leitende Frage. Aber bei solchen Versuchen verschwindet jedesmal der Wald vor lauter Bäumen, und es ist kein Wunder, daß z.B. die Begriffe 'Romantik' 
oder 'Barock' oder 'Realismus' sich unter der europäischen Perspektive so erweitern müssen, daß sie, eigentlich überhaupt nichts Spezifisches mehr besagen und am besten beseitigt würden. Mit dem Expressionismus könnte es leicht auch so passieren. Der synthetische Gehalt der Begriffe - schließlich ihr ein-

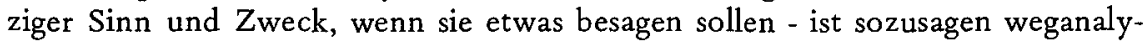
siert. Man weiß nichts mehr, weil man zuviel weiß, möchte ich pointiert sagen, .wenn das nicht eine mißverständliche und gefährliche Maxime für Studenten wäre! Anspruchsvoller ausgedrückt: Die Überdifferenzierung macht einen Abstraktionsgrad notwendig, bei dem sich - nach allen Regeln der Logik - der Umfang des Begriffs im gleichen Maß erweitert, wie sich sein Inhalt reduziert: je mehr Phänomene, Einzelerscheinungen er bezeichnen soll, umso weniger bestimmt kann natürlich sein Inhalt sein, umso ungenauer muß sein, was er bezeichnen soll. Nun hat der eben genannte Wellek eine ganz brauchbare Definition von 'Periodenbegriffen' oder 'Epochenbegriffen' versucht: Sie sind nämlich, so meint er, "Bezeichnungen für ganz bestimmte Normensysteme, welche jeweils die Literatur einer bestimmten Epoche beherrschen". Der Terminus 'Normensystem' - so Wellek - "ist eine zweckdienliche Bezeichnung für literarische Konventionen, Themen, philosophische Denkrichtungen, Stilmerkmale und dergl., während das Wort 'beherrschen' das Überwiegen einer Gruppe von Normen bezeichnet im Vergleich zum Überwiegen anderer Normen zu anderen Zeitabschnitten oder Perioden". 4 Nun muß man allerdings hinzufügen, $\mathrm{da} ß$ in einer 'Periode' oder 'Epoche' mehrere solcher 'Normensysteme' nebeneinander und gleichzeitig ihren Anspruch geltend machen können, manchmal in Konkurrenz zueinander, manchmal auch nicht unbedingt: man denke etwa an die verschiedenen Normenformationen in der Zeit Goethes: Aufklärung, Empfindsamkeit, Sturm und Drang, Klassik, Romantik, Biedermeier zum Beispiel - was ja keineswegs alles nacheinander weg kommt, sondern in unterschiedlichen Phasen zum Teil einigermaßen ineinander steckt. Und ob man von den Einheitsbegriffen - sagen wir - 'Klassik' oder 'Romantik', oder 'Frühromantik' oder 'Spätromantik' spricht, hängt wesentlich von der Perspektive und vom Kontext $\mathrm{ab}$, in dem man die geschichtlichen Phänomene betrachtet, und es kommt auf den Kulturzusammenhang an, von dem aus das Erkenntnisinteresse des Historikers geleitet ist. Gelegentlich können auch zunächst rein chronometrisch, chronologisch fungierende Zeitbezeichnungen zu synthetischen Epochenund Stileinheitsbegriffen aufsteigen. Das kann man etwa sehen an der Begriffsbildungsgeschichte, der Anreicherung der einfachen Bezeichnung 'Neunzehntes Jahrhundert'. Inzwischen besagt sie - je nach Perspektive und Art des Gebrauchs - erheblich mehr als reine Zeitabgrenzung; deutlicher noch wird das, wenn man das Fremdwort 'Dixneuvième' wählt mit dem ganzen Stilaroma, das daran haftet, wobei - nebenbei bemerkt - ein Vergleich zwischen der Bedeutungsfracht dieses Wortes im französischen und im deutschen historischen und historiographischen Kontext sehr aufschlußreich ist. Ähnliches läßt sich mit der Bezeichnung 'Siebzehntes Jahrhundert' beobachten, innerhalb dessen 'Barock' 
wieder ein besonderes Normensystem ist. Das 'Zwanzigste Jahrhundert' weiß noch nicht so recht, was aus ihm in begriffsgeschichtlicher Hinsicht wird. Eine ganze Reihe von Stileinheitsnamen innerhalb dieses Ganzen haben sich schon etabliert, etwa - wenn wir etliche Sonder-Ismen in der Malerei aus dem Spiel lassen - Naturalismus, Jugendstil, Symbolismus, Neuromantik, Impressionismus, Expressionismus, Neue Sachlichkeit - um einige wenigstens bis in die zwanziger Jahre zu nennen. Ja, und dann die 'Zwanziger Jahre' selbst, die gerade dabei sind, sich als Stil- und Periodenbegriff anzubieten.

Solche Vorüberlegungen mögen zur Frage des Themas überleiten, die ja ein wenig provozierend formuliert ist und schon fast eine nicht mehr weiterer Diskussion bedürftige These enthalten könnte: nämlich die, daß Expressionismus auf jeden Fall ein deutsches Phänomen sei. Aber es fragt sich, ob schon das richtig ist. Anders gewendet und im Anschluß an Welleks Vorstellung vom 'herrschenden Normensystem' kann man formulieren: Gibt es ein Normensystem mit dem Namen 'Expressionismus', das in einem bestimmten historischen Kontext, in einem bestimmten Kulturzusammenhang ebenso zureichend spezifisch wie auch zureichend weit ist, um eine Richtung und Spielart europäischer Moderne in Deutschland und in außerdeutschen Ländern zu 'beherrschen' und mit seinem Begriff zu bezeichnen? Oder gibt es nur ein wesentlich begrenzteres Normensystem 'Expressionismus', das, abgesehen von einigen außerdeutschen Einflüssen, die es aufgenommen hat, doch eigentlich nur die deutsche Situation betrifft, von ihr bestimmt wird und sie bestimmt? Die Fragen sind nicht neu, in der Zeit des Expressionismus tauchen sie schon auf, und auf einen internationalen Konnex legen viele Expressionisten selbst Wert.

Deutlicher als in der Literatur ist das im Bereich der Malerei und der Kunstkritik zu sehen. Man denke etwa an die Maler des 'Blauen Reiters' und der 'Brücke', man denke an den berühmten Almanach des 'Blauen Reiters', an die Ausstellungen und Programme des 'Sturms', an die wesentliche Rolle von Ausländern im Bereich der Kunsttheorie, etwa Kandinsky, um nur einen Namen zu nennen. Indessen ist nicht zufällig, daß viele Kunsthistoriker gerade ihn oder auch andere, seinerzeit moderne Maler wie etwa Franz Marc und verwandte, nicht in die Schublade 'Expressionismus' zwängen lassen wollen. Was den Beitrag Kandinskys, Marcs und ähnlicher zur Emanzipation, zur Reinigung der Form, der Formen vom Expressionismus trennt, ist eben das, was sie mit modernen, zum Ungegenständlichen tendierenden europäischen, insbesondere mit einigen führenden französischen Künstlern verbindet; und was sie mit denen verbindet, ist gerade das, was die europäische Avantgarde in der Malerei nicht einfach mit dem Expressionismus gemeinsam hat. Daß der Expressionismus eine 'typisch deutsche Angelegenheit' sei, ist die Überzeugung der meisten Kunsthistoriker. Wenn die Deutschen von Expression, von Ausdruck, Ausdruckskunst sprechen, meinen sie von Anfang an etwas anderes, als was die Franzosen mit dem Terminus 'expression' im Sinn gehabt hatten. Nachdem der Terminus etwa 1911 den Rhein überquert hatte, gab es den 'Expressionisten', 
der sich zwar vorerst selbst nicht so nannte, der aber - so hat es ein amerikanischer Forscher formuliert - "gave expression to emotions, feelings, the spiritual and psychological strivings of his time ... Moreover ... the creative process, universal and absolute, was itself the purpose and goal of art". 5 Man muß also, so meint dieser Gewährsmann, den ursprünglichen französischen und den späteren, von der deutschen Entwicklung her, bestimmten Gebrauch bei aller Gemeinsamkeit der modernen europäischen Kunst deutlich voneinander unterscheiden. Das ursprüngliche Element des Terminus bleibt erhalten - insofern ist der 'Expressionismus' im neueren Gebrauch des Wortes in der Kontinuität seiner Herkunft zu sehen -, aber dies ursprüngliche Element geht ein in ein Konzept von 'emotionalism', 'art of emotional expression'. In Deutschland gibt es auch den anderen Typus der Kunst, den der strengen und eher architektonisch-geometrischen Gestaltung, den ein anderer Kunstwissenschaftler: Werner Haftmann, einer der besten Kenner der modernen europäischen Kunst, dem 'elementaren Ausdruckswillen' des Expressionismus gegenüberstellt. ${ }^{6}$ In Hans von Marée sieht er einen entscheidenden Namen für den Beginn dieser Formtendenz im deutschen Bereich. Kandinsky ist dann eine Schlüsselfigur, und exemplarisch ist der Weg Franz Marcs zum Abstrakten oder Konstruktiven, zur Auflösung und Verwandlung des Sichtbaren ins Medium kristallischer Formen. Das 'Bauhaus', von dem ja gerade in letzter Zeit wieder im Zusammenhang mit seinem Gründer Walter Gropius häufiger die Rede war, spielt für die Förderung der Alternative zum Expressionismus oder in gewissem Sinne auch im Expressionismus eine produktive Rolle. Maler, die dafür beispielhaft sind und die Gropius an sein Institut holte, sind Paul Klee, der ja auch zum Freundeskreis des'Blauen Reiters'gehörte, dann Lyonel Feininger, Oskar Schlemmer und wieder Wassily Kandinsky. Man sieht übrigens hier - dies nebenbei - die Phasenverschiebung im Vergleich zur Literatur (Das Bauhaus beginnt erst 1918). Die Abstraktionen dieser Künstler beruhen auf einem neuen, einem - so Haftmann - "ganz tiefen Erlebnis der sichtbaren Natur". An zwei Beispielpaaren macht Haftmann den Unterschied von französischen und deutschen Formprinzipien in der modernen Kunst deutlich: George Braque 'Geige und Krug' - Franz Marc 'Tierschicksale'; Fernand Léger 'Drei Frauen' - Oskar Schlemmer 'Vierzehnergruppe'. In der französischen Gruppe - stellt Haftmann fest - "eine in sich geschlossene Architektur aus Ordnung und Form", in der deutschen "mehr 'Illustration', mehr dichterisches Gleichnis einer den ganzen Menschen umfassenden Welterfahrung". "Es gelang den Deutschen nicht, das Bild allein als ein in sich abgeschlossenes harmonikales Gebilde zu sehen ... Im Verhältnis zur französischen Malerei zeigt die deutsche Malerei einen ausgesprochen romantischen Charakter". ${ }^{7}$ Diese Beurteilung der. Lage ist einigermaßen exemplarisch für die Kunstwissenschaft. Sie gerät in gewisse Schwierigkeiten, wenn sie einige belgische, flämische, skandinavische Maler in ihre Betrachtung einbezieht, wie etwa Munch, Ensor, den späteren van Gogh, und da behilft sie sich dann, indem sie das deutsche Specifikum immerhin zu einem nordischen erweitert. 
Die Phasenverschiebung nach der anderen Seite, also in die Zeit vor irgendeinem literarischen Expressionismus, wollen wir wieder außer Betracht lassen: Mit dem Terminus des Nordischen verstärkt sich aber beinahe noch die Vorstellung einer fast qualvollen Ausdruckskunst, die ihre Schöpferkraft aus dem Leiden der Seele, aus der Tiefe des Abgründigen bezieht.

Expressionistische Architektur war lange Zeit kaum ein geläufiger Begriff, mit dem sich eine seriöse Vorstellung verband. Seit einigen Jahren gibt es eine ernstzunehmende Forschung, die ihren Gegenstand überhaupt erst einmal abgrenzen und plausibel machen mußte - woran denkt man schon, wenn man rasch sagen soll, was expressionistische Architektur ist? Wenn ich eine Reihe von Stichwörtern nenne, wird bei dem meisten den meisten nicht allzuviel einfallen: etwa 'Arbeitsrat für Kunst', Bruno Tauts 'Programm für die Architektur' (1918), Paul Scheerbarths Schrift 'Die Glasarchitektur' (1914), das 'Bauhaus', die Namen von Architekten und Lehrern der Architektur wie Finsterlin, Hablik, Gösch, Poelzig, Gropius, Mies van der Rohe, der eben schon genannte Bruno Taut sowie sein Bruder Max Taut. Es kommt auf einzelne Namen hier nicht besonders an. Ein Ausgangspunkt für so etwas wie eine Ideologie der expressionistischen Architektur ist die sogenannte 'Novembergruppe', nach der Novemberrevolution 1918 selbstbenannt, eine im Dezember 1918 in Berlin gegründete Künstlervereinigung, in der sich Architekten, Bildhauer, Maler, Dichter, Musiker und Regisseure zusammenfanden, um Kunst als Medium neuer Lebensformen zu fördern. Man hat von 'Utopisten' der Novembergruppe gesprochen, von ihrem 'technischen Mythologismus', 8 mit dem sie eine ideale Stadt für eine ideale Gesellschaft erträumten, und man hat mit Recht gemeint, die expressionistische Architektur habe 'ihren Höhepunkt' gehabt, "als sie noch nicht gebaut, sondern erst erträumt wurde", d.h. in der Zeit unmittelbar nach dem Ersten Weltkrieg. 9 Wieder sind das deutsche Phänomene, die nur vergleichsweise schwache Analogien in anderen europäischen Ländern haben. Sieht man auf die Grundlagen des technischen Konzepts dieser sozusagen expressionistischen Architektur, so bemerkt man eine Gleichsetzung von Konstruktion und Räumlichkeit: der Raum wird als etwas aufgefaßt, das mit der Form konstruiert wird, nicht mehr als etwas, worin die Form konstruiert wird. Diese Beobachtung, die Giulio Carlo Argan in einem Vortrag über ' $L$ 'architettura dell' espressionismo" mitgeteilt hat, scheint mir sehr zutreffend und spezifisch zu sein. ${ }^{10}$ Die Tatsache weist hin auf ein konstitutives und auf Emanzipation drängendes Element des Phantastischen, Poetischen, mindestens Idealistischen (im philosophischen Sinn des Worts) in einem Bereich, in dem Material und pragmatische Bedürnisse einen beträchtlicheren und legitimeren Widerstand leisten als im Bereich anderer Künste. Daraus entstehen die fast absurden Gebilde, in denen die Seele Material und Zweck überwältigen wollte. Sie sind nicht durch Zufall aus dem historischen Bewußtsein so gut wie verschwunden, Fachleute müssen sie mit Mühe und Not wieder entdecken.11 Ein Anschluß an die europäische Moderne war die expressionistische Architektur samt ihrer romantisch-utopischen 
Ideologie nicht. Die Produkte des Bauhauses, die ja eher bekannt sind und zum Teil bis in die Gegenwart aktuell geblieben sind, waren aber nicht mehr Expressionismus, sondern ein neuer ästhetischer Rationalismus, der eher Chancen hatte, die Grenzen der deutschen Provinz zu überschreiten.

Labil ist der Begriff 'expressionistische Musik'. Zwei durchaus verschiedene, zuweilen entgegengesetzte Tendenzen der Musik sind schon mit den Namen bezeichnet, die in Zusammenhang mit dem Expressionismus gebracht zu werden pflegen: Schönberg seit der I. Kammersinfonie op. 9 von 1906, dann Mahler, Berg, Webern, Bartók - um nur die wichtigsten zu nennen. Die Versuche, aus Tradition und überkommener Ästhetik auszubrechen und künstlerisch ins Unterbewußte, Irrationale und Transzendentale vorzudringen mit einer Radikalisierung der Tonsprache romantischer Musik, gehen in divergierende Richtungen: Abstraktion, Konstruktion, Atonalität, Symbolik, Karikatur, Groteske - Kennzeichen und Genera, die in der Musikwissenschaft als typisch für den Expressionismus bezeichnet werden, umfassen eine prekäre Form-Inhalt-Dialektik der musikgeschichtlichen Situation, in der die sogenannte expressionistische Musik angesiedelt ist. Die Tendenz zum Abstrakten, zur Reinigung der Form und die Tendenz zum Ausdruck geistiger und seelischer Anschauungen und Erfahrungen scheinen in der europäischen Perspektive aus unterschiedlichen Traditionen zu kommen. Die Entwicklung der romantischen Musik und ihrer Theorien zeigt, daß die anscheinend gegenläufigen Tendenzen sich nicht ausschließen, sondern wechselseitig fördern können. Dieses Problem betrifft natürlich auch die Literatur - davon sogleich -, aber die Musik hat ihre eigene europäische Produktions- und Rezeptionsgeschichte im neunzehnten und im zwanzigsten Jahrhundert, so daß Expressionistisches eher den Anschluß an moderne Initiativen im weiteren Bereich europäischer Musik finden und auf affinitive Situationen außerhalb Deutschlands und Österreichs stoßen konnte, auch wo viel deutsch-österreichische Seele, subjektives Empfinden, utopischer Humanismus, regionale. Folklore mit im Spiel waren: Weder allein europäische Moderne auf deutsch noch deutsche Seelenkrankheit - für die Musik jedenfalls ist die Formel unbrauchbar, die Alternative falsch, die sich aus einer Revue der internationalen Forschung zur Literatur anbieten könnte. Ob man für die moderne, den Expressionismus einschließende Musik mit einem Begriff des Antiaristotelischen weiterkommt, wie Jost Hermand vorgeschlagen hat, 12 bleibt allerdings auch sehr fraglich.

Was nun die Literatur betrifft, so legen die meisten Studien der letzten zwanzig Jahre mitsamt der Fülle neu ausgegrabenen und bearbeiteten Materials die alte Vermutung nahe, der Expressionismus sei denn doch, bei allen Anstößen von außen, von Frankreich zumal, aber auch aus einigen anderen westlichen und nordischen Ländern, eine überaus deutsche Angelegenheit und in der Tat Ausgeburt einer deutschen Krankheit - wenn man so will - und Malaise, in der Politisches und Psychisches sich wechselseitig bedingen und steigern. Die politische Seite der Sache ist oft genug abgehandelt worden, das 
gebrochene Verhältnis der Expressionisten zur sozialen und politischen Wirklichkeit. Nicht erst seit der Expressionismus-Debatte in der Moskauer Exilzeitschrift Das Wort hat man das chiliastische und utopische Menschheitspathos mit der deutschen politischen Situation und mit der Rolle der Intellektuellen darin in Zusammenhang gebracht. Die Gefahren, die sich daraus ergaben, die Auffüllbarkeit jenes zum Teil vitalen, vitalistischen, zum Teil geistig-mystischen, zum Teil religiösen, zum Teil sozialen, aktivistischen Raptus und eines Aufbruchs schlechthin mit politisch radikalen Ideologien, z.B. mit faschistisch-nazistischen oder kommunistischen, sehen viele bis heute eben als eine deutsche Misere an, als Krankheitssymptome einer kranken Wirklichkeit und eines kranken Verhältnisses zur Wirklichkeit, wofür in anderen Ländern die entsprechenden Voraussetzungen nicht existiert hätten. Weiter: Sowenig Nietzsches philosophische Analysen und Entwürfe eine begrenzte deutsche Angelegenheit sind, so entschieden scheint es doch eine mögliche Wirkung seiner Denkformen und -inhalte zu geben, die gerade eben den Erfahrungen der deutschen Expressionisten (und ja nicht nur der deutschen Expressionisten) entgegenkam und entsprechende Deutungen und Umdeutungen seiner Gedanken zeitigte. Ähnliches gilt auch für Freud, Bergson, Mach und andere. Wenn man die unzähligen Zitate in einem der jüngsten großen Bücher über Expressionismus studiert, mit denen das religiös-theologische Engagement der Expressionisten belegt werden soll, kann man kaum an die Thesen des Verfassers glauben, nach denen das 'Zerebrale', die 'zugespitzte Intellektualität', die 'Hirnlichkeit' eine hervorstechende Eigenschaft der Expressionisten sei, also eine Art versteckter Rationalität und Aufklärung im europäischen, im westlichen Stil.13 Der italienische Literaturwissenschaftler Ladislao Mittner hat schon in den sechziger Jahren geradezu modellhafte Analysen des Expressionismus im Sinne eines deutschen pathologischen Phänomens gegeben, die mehr oder weniger modifiziert in einen großen Teil der späteren Expressionismus-Kritik und -forschung eingegangen sind. Bemerkenswert ist, was in einem dieser Beiträge ${ }^{14}$ iiber Anfangs- und Endpunkte des Expressionismus im Hinblick auf Daten und exemplarische Dichtungen gesagt wird, etwa auch zu der politischen 'Klammer', die von den zaristischen Judenpogromen einerseits und Hitlers Judenverfolgung andererseits gebildet wird: unter dem Einfluß der ersteren kam es' zum Gegensatz von westlichem Aktivismus und orientalischem Mystizismus, in der Folge der letzteren zum Ende der deutsch-jüdischen Literatur in Deutschland. Keiner der großen Schriftsteller der Epoche des Expressionismus, so meint Mittner, hat dem Expressionismus angehört. Er war in erster Linie Kunst- und Lebensstil, also Technik und Programm. Die 'wahren' und 'reinen' Expressionisten sind im ganzen Figuren dritten und vierten Ranges. Manche haben kurze Zeit dem Expressionismus nahegestanden, so daß auch Nicht- oder zukünftige Antiexpressionisten $\mathrm{zu}$ seiner Entwicklung beitrugen. Mit Ausnahme vielleicht allein des Zaubers der schönsten Gedichte Trakls hat der Expressionismus keine Meisterwerke hervorgebracht. Auch in einem anderen ausgezeichneten Beitrag 
zum Expressionismus ${ }^{15}$ legt Mittner ein solch entschiedenes und exklusives Bekenntnis zu Trakl ab. Der Expressionismus hat im übrigen die Phase der Negativität nicht überwunden, nicht so sehr aus äußeren Gründen (wie Zensur, Krieg etc.), sondern weil ihm eine klare Vorstellung der politisch-sozialen Richtung seiner Rebellion fehlte.

Wenn man nach den Gründen für das "fast totale Scheitern des Expressionismus als politisch-soziale Bewegung" suche, müsse man in die Zeit zurückgehen, in der die Expressionisten sich noch nicht so nannten, sondern nur als Anhänger der Gruppen der Neopathetiker, der Äternisten und der Aktivisten erschienen. Es gibt in der Unklarheit über die konkrete Form einer Erneuerung eine 'concordia discors' dieser Gruppen. "Die einen wie die anderen strebten danach, das reine Wesen des Menschen, den Nackten Menschen zu erreichen, und das führte sie unbewußt dazu, ein politisch-soziales Problem in ein metaphysisches, genauer in ein kosmisches zu verwandeln". Ein entscheidender Schlüsselbegriff, bei dem auch die kritische Reflexion über den Expressionismus anzusetzen hat, ist der des 'Geistes'. 'Die suggestive Polyvalenz des Begriffes 'Geist' erklärt zum großen Teil den Erfolg der gesamten expressionistischen Bewegung, aber auch das Scheitern ihres politisch-sozialen Programms". Nun ist das bisher noch nichts Neues, auch nicht im Vergleich mit Mittners ein Jahr früher erschienenem Aufsatz. Aber dann kommen einige Bestimmungen, die uber den damaligen stand der Erkenntnis hinausführten. Da ist zunächst die Vorstellung von der Gewaltlosigkeit, die immer und so auch im Expressionismus in seiner historisch-konkreten Weise zu Aporien führt. Aus der Erfahrung des Krieges und seiner Verdammung ergab sich folgerichtig eine Verdammung von "Gewaltanwendung überhaupt . . . welches Ziel sie auch immer habe. Da die Macht das Böse an sich war, mußte man notwendigerweise deren Gegenteil, die UnMacht vertreten, die man aber als aktive Macht empfand, insofern sie sich in dem entschiedenen, heroischen passiven Widerstand gegen die gewaltübende Macht entfaltet". Eine Betrachtung der dichterischen Realisierungen solcher Ideen zeigt, daß die "revolutionäre Tat ... durch einen Aufruf der Affekte geschwächt" wird, "der mit der Tat selbst unvereinbar scheint". Bei einigen, die Gewaltlosigkeit propagierten, spielten franziskanische oder orientalische Gedanken eine Rolle. In Mythisierungen des Revolutionsideals; wie etwa bei. Toller und Rubiner, wird es 'enthistorisiert' und 'in utopische Ferne' projiziert. Der Vater-Sohn-Konflikt im Expressionismus ist mit der 'expressionistischen Regression' eng verknüpft. Der Vater ist der oft erste und einzige Vertreter einer politisch-sozialen Ordnung, gegen die man sich richtet. Es ist kein Zufall, daß der "Konflikt zwischen Kapital und Arbeit oft in indirekter oder absichtlich getarnter Form als ein internes Geschehen im Hause des Kapitalisten behandelt" wird, "als ein 'unvermeidbarer' Generationskonflikt, als ein Konflikt zwischen den Reichen und ihren Söhnen, die die Väter verdammen und bekämpfen". Vatermord, wo er als Absicht oder Versuch vorkommt, wird im übrigen "mehr aus persönlichen Motiven vollzogen oder wenigstens geplant, 
als aus eigentlich sozialen". Die "Rebellion gegen die Gesellschaft" beschränkt sich "auf einen symbolischen Akt, der innerhalb der vier häuslichen Wände oder im Konferenzzimmer des Gymnasiums verborgen bleibt und niemals zur öffentlichen Diskussion gelangt. Der neue Tyrannenmörder ist daher ein etwas lächerlicher Brutus, der unterm Ödipuskomplex, das heißt unter einer ziemlich schwerwiegenden Form von Infantilismus leidet . . . Mit dem Expressionismus beansprucht der rebellische Student für sich die Freiheit: aber nicht nur oder nicht so sehr die politisch-soziale, als vielmehr die noch verweigerte sexuelle Freiheit". Der Hinweis auf den Ödipuskomplex der Expressionisten ist nicht neu; Mittner weist aber zum erstenmal hin auf die "starke, ja vorherrschende weibliche Komponente ..., nämlich die Bindung an die Mutter". Und diese Figur der Mutter hat oft die "düstere und unheimliche Gestalt der großen Erdmutter, der Erzeugerin und Verschlingerin der eigenen Kinder". Im Kontext dieser Tatsache und des spezifischen 'Infantilismus' der Expressionisten muß man, wo es vorkommt, das Thema des Kampfes zwischen Mann und Frau sehen. Aus den Träumen des gescheiterten. Helden, des Jünglings, "der zur Tatenlosigkeit verdammt ist" und sich suggeriert, sich mit vorgestellten "Grausamkeiten in der sozialen oder wenigstens in der geschlechtlichen Wirklichkeit durchzusetzen", wird die "Gestalt des velleitären Tyrannen, des Tyrannen in der Einbildung geboren. Diese Gestalt muß wenigstens teilweise in die Realität eintreten: der introvertierte Masochismus erzeugt sein untrennbares extrovertiertes Komplement, den Sadismus". Die Konvertibilität von Subjekt und Objekt des Mordes, die "Austauschbarkeit des Opfers und des Mörders" in einigen expressionistischen Dichtungen hängt aufs engste damit zusammen. Ebenso ist die 'Figur des bösen Magiers', die den expressionistischen Film beherrscht, von solchen Überlegungen her verständlich. Das alles kennzeichnet den 'Ungeist' des Expressionismus. Am Schluß rückt Mittner indessen seine entschiedene Kritik ins angemessene Verhältnis zu den positiven Aspekten des expressionistischen 'Geistes', des 'echten Geistes', den diese Bewegung denn doch auch vertrat. "Es wäre ungerecht", den "künstlerischen Experimentalismus" des Expressionismus "en bloc als dilettantischen Avantgardismus abzutun ... Der 'Geist' ist zwar nie eigentlich 'marschiert' (damit spielt Mittner auf ein Wort Kurt Hillers an, das er seinem Essay als - ironisches - Motto vorangesetzt hat), weder mit einer Nation, noch mit einer Partei; er hat aber, alles in allem, sich selbst nicht verraten: er versuchte - mit einer Art verzweifelter Besessenheit -, sich und die Welt zu erhellen". Man sieht: das ist durchaus deutsche Pathographie, Pathographie einer deutschen Variante von Moderne (oder auch Nicht-Moderne).

Nun gibt es seither nicht nur eine breitere internationale Forschung, die sich mit diesem mehr oder weniger leidigen oder auch imponierenden deutschen Expressionismus befaßt: in England, Frankreich, Italien, USA, den Niederlanden, Belgien, Jugoslawien, Polen, Ungarn, Rumänien, Rußland, Südamerika, Skandinavien. Die Außenperspektive ist da für so manchen deutschen wissen- 
schaftlichen Tiefsinn oft wohltuend ernüchternd und erleichtert Distanz. Außerdem lernt man manches über wechselseitige Einwirkungen, Einflüsse, katalysatorische Effekte. Was aber interessanter ist: die Forschung in den verschiedenen Ländern ist - zuweilen bei Gelegenheit der Beschäftigung mit dem deutschen Expressionismus, zuweilen auch bei dem Geschäft der Historiographie der eigenen Nationalliteratur - fast überall auch auf einen eigenen Expressionismus gestoßen. Von mehr oder weniger schwachen Analogien hatte man für den einen oder anderen Fall gelegentlich gewußt. Daß es von Deutschland aus und nach Deutschland hin Anregungen gab, das konnte man gewiß bei den Expressionisten und nichtexpressionistischen Zeitgenossen selbst schon lesen. Einen internationalen Aspekt des Phänomens Expressionismus brachte zum ersten Mal nicht nur den Spezialisten ein Sammelband zahlreicher Essays zum Bewußsein mit dem Titel Expressionism as an International Literary Phenomenon. ${ }^{16}$ Wieweit geht nun tatsächlich die Gemeinsamkeit, nach der der Titel klingt? Der Herausgeber, Ulrich Weisstein, Professor für Comparative Literature an der University of Indiana in Bloomington, ist - obgleich er den Band mit dem Thema initiiert hat - vorsichtig und zurückhaltend in der Voraussetzung eines durch die Länder einheitlichen Expressionismus. Wie ein paar andere Forscher in den letzten Jahren auch, hilft er sich aus einigen Verlegenheiten mit der Annahme mehrerer Expressionismen: "What we have, in fact, is not one Expressionism but a number of loosely connected and subtly - or not so subtly - differentiated Expressionisms".17 Das ist bei einer bestimmten Art des Beharrens auf Eindeutigkeiten in Epochenbegriffen eine nicht unbekannte Reaktion - ich erinnere an einige Eingangsüberlegungen -,wie man ja auch schon verschiedene Romantiken, 'Romanticisms', statt einer Romantik vorgeschlagen hat. Trotz aller Vorbehalte und Restriktionen im Hinblick auf die Definierbarkeit des Expressionismus hält auch Weisstein, der die Expressionismen vergleichen kann und verglichen hat, an einer entschiedenen Abgrenzung gegenüber anderen Strömungen und Tendenzen der modernen Kunst und Literatur fest. Er wehrt sich energisch gegen Sätze Gottfried Benns von 1933, die er 1955 wiederholt hatte und in denen er Futurismus, Kubismus, Surrealismus als Synonyme für Expressionismus gebrauchte. In Deutschland und in den angelsächsischen Ländern unterscheidet man - nach Weisstein glücklicherweise - zwischen diesen verschiedenen Tendenzen, im Gegensatz zur Romania und Osteuropa. Da herrschen allgemeinere Bezeichnungen vor wie 'Modernismus', 'Avantgarde', 'progressive' Kunst und Literatur. In den osteuropäischen Ländern bedeuten die Namen der modernistischen Richtungen oft etwas ganz anderes als in Westeuropa. Z.B. hat der russische Futurismus außer der Bezeichnung nichts gemein mit der Initiative Marinettis und seiner Freunde. Wichtig zu bedenken für die osteuropäischen Länder ist zudem der enorme "Nachholbedarf, "the urgent need", seit ihrer "sociopolitical emancipation for catching up with Central European developments from ca. 1870 on. Hence the near-simultaneity, observed in Hungary and elsewhere of Naturalism, Expressionism and Surrealism". Der Behauptung 
des gescheiten ungarischen Literaturwissenschaftlers und Philosophen György Mihály Vajda ist nicht zuzustimmen, nach der der Expressionismus einen der charakteristischen Züge aller romantisch-modernistischen Tendenzen bezeichnet: "For such a view would seem to deny, by implication, the modernity of the classical vein inherent in Cubism, De Stijl, Constructivism, Suprematism and even Vorticism".18 Davon abgesehen - oder eigentlich in der Konsequenz dieser Überlegungen - ist davor zu warnen, 'Expressionismus' und 'abstrakte Kunst' durcheinander zu werfen, was immer wieder geschieht, nicht zuletzt auf Grund einer Fehlinterpretation von Worringers Begriff der Abstraktion. (Wilhelm Worringer hatte ja mit seiner berühmten, vor einigen Jahren wiedergedruckten Dissertation von 1908 mit dem Titel Abstraktion und Einfühlung. Ein Beitrag zur Stilpsychologie eines der theoretischen Grundbücher für eine expressionistische Ästhetik geliefert, neben Kandinskys Über das Geistige in der Kunst (1910) ). Der Expressionismus hat zwar die Tendenz zum Ungegenständlichen, aber er hat "nothing in common with the controlled, disciplined and, at times serene 'Ausdruckskunst' of Neo-Plasticism or Suprematism".19 Expressionismus ist entschieden 'content-oriented'. Expressionismus ist anthropozentrische Kunst. Landschaft, Tiere, Technisches sind vermenschlicht, im Unterschied etwa zum Futurismus und Vortizismus. Auch ist der Expressionismus nicht so auf Simultaneität aus wie etwa Kubismus und Futurismus. Die bevorzugten Reiche des Expressionismus waren entweder 'cosmic' oder 'broadly humanitarian'. Die Expressionisten richten sich auf das Wesen, 'to essences', ohne die phänomenale Welt, die Welt der Erscheinungen loszuwerden. In der Psychologie zeigt der Expressionismus eher Affinitäten zu einer Art von Konzept wie dem Jungs, während Freud für den Surrealismus eine angemessenere Anschauung bezeichnet.

Vorhin habe ich gesagt, daß in den letzten Jahren die Forschung in den meisten europäischen Ländern nach Expressionistischem in der eigenen Literatur gefahndet habe. Sieht man die große Zahl von Veröffentlichungen, sieht man die Texte an, mit denen so etwas wie Expressionismus belegt werden soll, so ergibt sich ein mehrdeutiges Bild, bei dem man genau unterscheiden muß: Nicht zur Debatte - um es zu wiederholen - können hier Namen und literarische Produkte aus nichtdeutschen Ländern stehen, die vom deutschen Expressionismus rezipiert worden sind und die - oft auf sehr verschiedene Weise - zu seiner Ausbildung beigetragen haben, die für die expressionistische Ästhetik und Poetik in allen Gattungen in Theorie und Praxis und für politische, philosophische, soziologische, religiöse Ideen des Expressionismus wirksam geworden sind. Nirgendwo ist der deutsche Expressionismus eine Summe aus solchen Elementen des Einflusses, er läßt sich nicht darauf reduzieren, und was aus dem Ausland kam, kann man nicht gewissermaßen für den deutschen Expressionismus verantwortlich machen. Ob man an Strindberg denkt, an Whitman, Verhaeren, Rimbaud, Baudelaire, um eine beträchtliche und zahlreiche Namenreihe anzudeuten, die mit diesen Genannten ja erst angefangen ist und hier nicht vollständig aufge- 
zählt werden muß, ob man von Futurismus, Imagismus, Vortizismus, frühestem Surrealismus redet, von politischen Ereignissen außerhalb Deutschlands - alles das erklärt nicht zureichend das deutsche Phänomen des Expressionismus. Bedeutsamer, interessanter für unser Thema ist schon die umgekehrte Perspektive, nämlich die Wirkung des deutschen Expressionismus auf die Literatur außerdeutscher Länder. Für einige Länder gilt - fast jedenfalls - 'Fehlanzeige', z.B. für Frankreich. Es gibt da zwar ein relativ spätes Interesse an dieser dubiosen Variante einer sich modern und avantgardistisch gebärdenden fortschrittlichen Kunst. Einige Zeitschriften berichteten darüber, meist kritisch, oft eher ablehnend: die Nouvelle Revue Française, der Mercure de France, die Zeitschrift Clarté, die Revue Rhénane und ein paar andere Journale. Im Grunde wird da der Expressionismus als mindestens rückständig oder vielmehr verspätet empfunden. Und das ist aus der französischen Perspektive durchaus begreiflich: Es gab ja kein Vakuum für den Expressionismus, muß man wohl hinzufügen: Man hatte in Literatur und bildender Kunst längst seine eigene Avantgarde, der gegenüber der deutsche Expressionismus als sekundär und in gewissem Sinne als provinziell erscheinen mußte. Die fast emphatische Ignorierung "méconnaissance du mouvement expressionniste" 20 geht so weit, daß man Expressionisten, selbst wenn man sie schätzt, einfach nicht Expressionisten nennt. Immerhin konnte noch 1954 André Breton an Lotte Eisner, anläßlich eines Essays, den sie über den expressionistischen Film verfaßt und ihm geschickt hatte, schreiben: "Ce que vous révélez des intentions profondes de l'expressionnisme est absolument essentiel. J'enrage à penser que cela a été si bien occulté dans ce pays".21 Auch in England ist das Interesse nicht übertrieben groß. Als es eigentlich hätte aktualisiert werden können, hatte man seinen 'Vorticism', hatte man seine eigene Zeitschrift Blast: Review of the Great English Vortex. Die weltanschauliche Brunst, das Röhren des Absoluten bei den deutschen Expressionisten war selbst für exzentrische, skurille, absurde, antimechanistische Engländer nichts. Vorticism aber ist definitiv nicht einfach Expressionismus auf Englisch! - Die Italiener hatten ihren Futurismus. Auch österreichische Avantgarde ist ein Spezifikum. Aber hier gibt es doch einiges eher Verwandte, wenn auch die Österreicher die Strapazen fanatischer und schwitzender Weltanschauungen mit allzu viel Metaphysik nur sehr vorübergehend auf sich zu nehmen belieben. Aber der österreichisch-deutsche Sprachbereich läßt sich zur Zeit des Expressionismus weniger getrennt betrachten als vielleicht später: Kokoschka, Werfel, Ehrenstein, Däubler, Trakl, Raoul Hausmann, Ernst Weiss, die jeder mit dem Expressionismus irgendwie in Verbindung bringt, sind schließlich Österreicher. - Weniger selbstverständlich sind schon expressionistisch anmutende Erscheinungen in anderen Ländern des Gebiets, das eine vor kurzem erschienene Essaysammlung 'europäisches Zwischenfeld' nennt: Expressionismus, Expressionisten im europäischen Zwischenfeld:22 Österreicher sind damit gemeint - davon war gerade die Rede -, dann Ungarn, Tschechen, Slowaken, Südslawen, Rumänen. Für alle einzelnen Regionen werden auch sonst in der 
Forschung Namen von Schriftstellern, auch von Zeitschriften genannt, die Zeugen sind für Tendenzen, die dem deutschen Expressionismus verwandt sind: ich will hier kein großes 'Name-dropping' veranstalten: das behält man nicht im Kopf, wenn man die Dinge und Personen nicht ohnehin schon kennt. Das macht aber auch nichts. Fast für alle nationalen Varianten kann man jedenfalls konstatieren, was der jugoslawische Germanist Viktor Žmegač für den 'kroatischen Expressionismus', wenn immer man ihn so nennen kann, festgestellt hat, daß nämlich eine 'Ausdruckskunst' wie z.B. die von Antun Branko Šimić - um den bedeutendsten Namen im kroatischen Bereich zu nennen - nicht "Spiegelung eines bestimmten 'Einflusses', sondern 'Durchbruch eines spezifischen Kunstdenkens' " sei, "das in der Folge gleichartiger historischer Impulse als geschichtlich synchroner Vorgang zu deuten ist". Nur partiell handele es sich da und andernorts um das Ergebnis einer Faszination von außen, nämlich von Deutschland - auch darum-, vielmehr weitgehend um die Teilhabe an einer allgemeinen europäischen sozialgeschichtlichen und künstlerischen Situation, so verschieden die Voraussetzungen und Bedingungen in einzelnen Ländern gewesen sein mochten. Das werde verständlicher, wenn man den Charakter der spontanen Plötzlichkeit als Signum des Expressionismus nicht übertreibe, sondern ihn - im gesamteuropäischen Kontext - als Element einer integralen geschichtlichen Reihe von Tendenzen und Wandlungen sehe. Jedenfalls setzt eine Rezeption von Motiven der europäischen Moderne und im besonderen des deutschen Expressionismus eine Affinität, eine Prädisposition in den Kultur- und Literatursituationen in den verschiedenen Nationen voraus. Je genauer man das Einzelne ansieht, etwa auch die Berichte über die avantgardistischen Zeitschriften in Kroatien, Polen, Rumänien, Ungarn, Bulgarien, umso mehr verflüchtigt sich der Expressionismus, den es da geben soll, zu einem mixtum compositum recht verschiedener modernistischer Tendenzen und Positionen. Diverse Ismen, die mehr oder weniger vergleichbaren westeuropäischen Phänomenen entsprechen, lösen sich rasch ab und sind häufig miteinander verschränkt. Auffallend ist außerdem, $d a ß$ in einigen Ländern avantgardistische Tendenzen, Experimente, 'Aktionen', die dem Expressionismus oder auch dem Futurismus und Surrealismus ähnlich sind, mit Verspätung auftreten und länger in die zwanziger Jahre hinein wirksam bleiben als in Deutschland und Westeuropa. Anzumerken ist die vielfach ganz andere und positive Rolle, die Traditionen der eigenen Literaturen der osteuropäischen Länder, auch folkloristische Traditionen in den avantgardistischen Versuchen spielen. Wie im westlichen - und das heißt dann doch im wesentlichen immer: im deutschen - Expressionismus haben Schriftsteller von einiger Bedeutung nur so etwas wie eine expressionistische Phase durchschritten, um dann zu ganz anderen Positionen zu kommen. Manche tendieren mit der Zeit mehr zum Abstrakten, andere verbinden ihre literarische Arbeit entschieden mit Zielen politischer Aktion. Das Wort Expressionismus ist jedenfalls für die Länder des 'europäischen Zwischenfeldes' in hörbare Anführungszeichen zu setzen. Es handelt sich allenfalls um 'expressionistoide' 
Phänomene im 'Magnetfeld' des deutschen Expressionismus, wie es der Kroate Gustav Krkleと ausdrückt. Das aber gibt es immerhin. Es hängt gewiß auch mit, wenn auch noch so disparat gewordenen, Gemeinsamkeiten und Traditionen im deutschösterreichisch-donaumonarchischen Großraum zusammen. In anderen Gebieten beruht die Faszination, die dieser merkwürdige deutsche Raptus 'Expressionismus' ausübt, weniger auf verwandten psychischen, politischen, historischen Verwandtschaften. Der Russe Konstantin Fedin etwa war während des Ersten Weltkriegs in Deutschland interniert, und da machte ihm der Kreis um die Zeitschrift Die Aktion besonderen Eindruck. Aber man hat mit Recht festgestellt, sein humanistischer Idealismus und seine Gesellschaftskritik seien in dieser seiner vom Expressionismus beeinflußten Phase durchaus individuell und privat geblieben. ${ }^{24}$ In Lateinamerika, insbesondere in Mexiko und Argentinien (auch in Uruguay und Venezuela) lassen sich in der Zeit von ca. 1915 bis 1940 expressionistische Symptome ausmachen, wieder initiiert vom deutschen Expressionismus, den einige lateinamerikanische Dichter und Literaten in Deutschland kennengelernt oder den deutsche importiert hatten. Wie wenig konkret das aber bei genauerer Analyse ist, zeigt die geschickte, fast pfiffige Formulierung in der jüngsten Studie zu diesem Bereich: es handele sich um einen 'unannounced expressionism', also um einen, der sich nirgendwo als solchen bezeichnet und auch nicht entsprechende theoretische Manifestationen und Programme aufzuweisen hat. 25 'Irrationalismus', 'Deformierung' der Wirklichkeit, die da zu sehen sind, bleiben in der Tat allzu unspezifisch. Auseinandergesetzt hat man sich mit dem deutschen Expressionismus gewiß auch sonst vielerorts, auch schon früh in Japan und China. Manchmal ist recht merkwürdig, was und wer da zufällig Anstöße gebracht hat - eine lange, ganz interessante Geschichte für sich. Unter den Dramatikern sind es übrigens fast immer Georg Kaiser, dann mit Abstand danach - Hasenclever und Toller, die katalysatorisch auf eigene Prädispositionen in nichtdeutschen Ländern gewirkt haben, z.B. in den USA: Besonders dazu gibt es ja auch in jüngster Zeit wieder einige umfassendere Untersuchungen. Abgesehen von gewissen Theatergruppen steht da der Name O'Neill immer im Vordergrund.

Zieht man aus alledem die Summe, so bleibt bei allen Osmosen, Analogien, Affinitäten moderner, modernistischer Ansätze, Experimente, Tendenzen außerhalb Deutschlands, die nicht zureichend mit anderen Ismus-Begriffen der europäischen Moderne beschreibbar sind, der Expressionismus denn doch ein deutsches Abenteuer, ein deutscher Versuch, auf das Niveau der westlichen Avantgarde zu gelangen, den Aufbruch in eine neue Ära, in eine neue Zukunft zu veranstalten - bis hin zu den Anfängen des Dadaismus in Zürich, die in ihrer Antithese gegen den Expressionismus wohl doch zunächst durchaus im Zusammenhang mit dem Expressionismus gesehen werden müssen. Gewiß gibt es eine Art Polygenese moderner Initiativen in der Kunst und Literatur verschiedener Länder, etwa in Osteuropa, einigen west- und nordeuropäischen oder auch lateinamerikanischen Staaten, wie wir gesehen haben, aber gerade soweit der 
Stilbegriff 'expressionistisch' Konkretes und Spezifisches trifft, handelt es sich um sekundäre Phänomene, die fast immer ihre Herkunft aus der deutschen Szene kennen und aktualisieren. Prüft man die Skala der Anwendungsvorschläge oder der Anwendungspraktiken für den Terminus 'Expressionismus'; wenn man die gesamte Forschung einbezieht, so scheint sich folgende communis opinio, ausgesprochen und häufiger fast unausgesprochen, als Umriß eines 'Normensystems' - um den Wellekschen Begriff hier wieder zu gebrauchen - herauszubilden: Expressionismus ist antimimetisch, er deformiert die Wirklichkeit, neigt im Vergleich zu aller irgendwie noch 'realistisch' zu nennenden Darstellung zur Abstraktion, zur Priorität von formalen Zeichen für seelische und geistige Gehalte gegenüber dem Arsenal von Gestalten aus einer empirischen Welt. Das verbindet ihn mit der europäischen Kunstmoderne, an die er sich im Prinzip anschließen will und anschließt: also insofern durchaus europäische Moderne auf deutsch. Aber im Gegensatz oder wenigstens im Unterschied zu anderen Varianten moderner, gleichfalls mehr oder weniger zum Abstrakten neigender Kunst gerät der Expressionismus stärker in eine dialektische Spannung von 'reiner' Form und greifbar gemachtem Inhalt. 'Realismus', wie weit auch immer verstanden, kann nicht Expressionismus sein. Abstrakte Kunst, reine Kombination von Formen ist ebensowenig expressionistisch. Das Besondere des Expressionismus scheint also zu sein, daß die verbreitete europäische Tendenz zum Abstrakten in der modernen Kunst und Literatur verbunden wird mit einer fast besessenen Absicht, das soeben von alten. Inhalten befreite 'Abstrakte', die aufs Formale reduzierten Strukturen wieder zum Medium eines wenn auch noch so vage bestimmten oder unbestimmten Inhalts seelischer und geistiger Realitäten zu machen. Diese Art von ebenso eindrucksvoller wie manchmal verkrampfter Form-Inhalt-Dialektik unterscheidet offenbar den Expressionismus von anderen, prima vista mindestens in formalen Phänomenen verwandt aussehenden (und ja auch tatsächlich verwandten) Versuchen in der künstlerischen und literarischen europäischen Avantgarde. Die zuweilen eher instinktive als genau begründete Abneigung kenntnisreicher Forscher, den Expressionismus in einem Atemzug mit den Varianten abstrakter Kunst zu nennen, anderseits diese Varianten einem allzu habgierigen Expressionismus-Begriff einzuverleiben, hat hier wohl ihren guten und richtigen Grund. Was die 'Inhalte' betrifft, auf die sich der Expressionismus richtet: das Religiöse, Politische, Soziale, Antibürgerliche, Menschheitliche, Geistige, 'Seelische', Sinnliche, Vitalistische und so weiter - so tun sich da die Forscher leichter, sich zu einigen und auch das Gegensätzliche als Element eines widerspruchsvollen und doch erstaunlich einheitlichen Ganzen gelten zu lassen, das sich verhältnismäßig einfach von anderen Regionen der europäischen Moderne abgrenzen läßt. Die spezifischen deutschen Inhalte und Gehalte freilich könnten die Vorstellung vom Expressionismus als einer deutschen politisch, sozial- und geistesgeschichtlich bedingten 'Seelenkrankheit' nahelegen, wenn dergleichen schöne Formeln nicht allenfalls als Reizwörter für Essaythemen, aber nicht allzu sehr für seriöse historische Erkenntnis taugen würden und wenn 
nicht einige der deutschen Leiden denn doch am Ende auch Varianten europäischer Leiden der Epoche - im weiten Sinn - wären. 26

\section{Anmerkungen}

1 Wolfgang Rothe, Der Expressionismus. Theologische, soziologische und anthropologische Aspekte der Literatur (Das Abendland, N.F. 9), Frankfurt a.M. 1977, S. 11.

2 Vgl. dazu u.a. Richard Brinkmann, "Gedanken über einige Kategorien der Literaturgeschichtsschreibung. Anläßlich der ersten beiden Bände von Friedrich Sengles Biedermeierzeit", Euphorion 69 (1975), S. 41-68.

3 René Wellek, "Der Barockbegriff in der Literaturwissenschaft", Grundbegriffe der Literaturkritik, Stuttgart-Berlin-Köln-Mainz 1965, S. 57-94. - Ders., "Der Begriff der Romantik in der Literaturgeschichte", ebd. S. 95-143. - Ders., "Noch einmal: Die Romantik", ebd. S. 144-160. - Ders., "Der Realismusbegriff in der Literaturwissenschaft", ebd. S. 161-182.

4 René Wellek, Grundbegriffe der Literaturkritik (s. Anm. 3), S. 96.

5 Donald E. Gordon, "On the Origin of the Word 'Expressionism" ", Journal of the Warburg and Courtauld Institutes 29 (1966), S. 368-385.

6 Werner Haftmann, "Maß und Form in der deutschen modernen Malerei", Der deutsche Expressionismus. Formen und Gestalten, hrsg. von Hans Steffen, Göttingen 1965, S. 226-249.

7 A.a.O., S. 248 f.

8 Giulio Carlo Argan, "L'architettura dell' espressionismo", Bilancio dell' espressionismo, Firenze 1965, S. 94-108.

9 Wolfgang Pehnt, "Gewißheit des Wunders. Der Expressionismus in der Architektur", Das Kunstwerk 17, H. 9 (1964), S. 2-11.

10 Siehe Anm. 8.

11 Vgl. die Architektur in expressionistischen Filmen wie z.B. Das Kabinett des Doktor Caligari.

12 Jost Hermand, "Expressionism and Music", Expressionism Reconsidered. Relationships and Affinities, ed. by Gertrud Bauer-Pickar and Karl Eugene Webb (Houston German Studies 1), München 1979, S. 58-73.

13 Siehe Anm. 1.

14 Ladislao Mittner, "L'espressionismo fra l'impressionismo e la 'Neue Sachlichkeit': frattura e continuitá", StudiGerm. 2 (1964), H. 2, S. 37-82 und H. 3, S. 38-75.

15 Ladislao Mittner, "L'espressionismo letterario", Bilancio dell' espressionismo, Firenze 1965, S. 11-29. 
16 Expressionism as an International Literary Phenomenon. 21 Essays and a Bibliography, ed. by Ulrich Weisstein (A Comparative History of Literatures in European Languages 1), Paris, Budapest 1973.

17 A.a.O., S. 17.

18 A.a.O., S. 21.

19 A.a.O., S. 23.

20 Lionel Richard, "Sur l'expressionnisme allemand et sa réception critique en France de 1910 à 1925", Arcadia 9 (1974), S. 266-289.

21 A.a.O., S. 286.

22 'Expressionismus' im europäischen Zwischenfeld, hrsg. von Zoran Konstantinović (Innsbrucker Beiträge zur Kulturwissenschaft, Sonderheft 43), Innsbruck 1978.

23 Viktor Žmegac, "Antun Branko Simić als Lyriker. Ein Beitrag zur Erforschung des kroatischen 'Expressionismus' ', Die Welt der Slaven 3 (1958), S. 151-165, hier S. 165.

24 B. Hiller, "Konstantin Fedin und der deutsche Expressionismus", Zeitschrift für Slawistik 10, H. 1 (1965), S. 35-49.

25 Naomi Lindstrom, Literary Expressionism in Argentina: The Presentation of Incoherence, Arizona 1977.

26 Der vorstehende Essay ist aus einem Vortrag an den Universitäten London und Cambridge entstanden.

Im übrigen sei verwiesen auf den soeben erschienenen umfassenden Forschungsbericht des Verfassers: Expressionismus. Internationale Forschung zu einem internationalen Phänomen, Sonderband der Deutschen Vierteljahrsschrift für Literaturwissenschaft und Geistesgeschichte, Stuttgart 1980. Einzelnes daraus ist hier übernommen. 